\title{
Analysis on Structural and Chemical Behavior with Cycles in PRAM
}

\author{
Kyuman Hwang, * Jong-bong Park,** Junsoo Bae, * Kwangho Park,* Seongbu Jeon,* Juhyeon Ahn,* \\ Seoksik Kim,* Gitae Jeong, ${ }^{* * *}$ Changjin Kang, ${ }^{*}$ Chilhee Chung**** \\ *R\&D Fab Operation Team, ***New Memory Lab, ****Semiconductor Research Center \\ Samsung Electronics Co., Ltd. San \#16, Banwol-Dong, Hwaseong-City, Gyeonggi-Do 445-701, \\ Korea, km0323.hwang@samsung.com \\ **Samsung Advanced Institute of Technology, San \#24, Nongseo-Dong, Giheung-Gu, Yongin-City, \\ Gyeonggi-Do 446-711, Korea
}

As a promising next generation non-volatile memory, PRAM (Phase Change Random Access Memory) faces entering the marketplace. However, the compositions of GST (GeSbTe), the core storage element in PRAM, is known to gradually change with programming cycles [1], so the accurate and correct analysis on the GST is important in order to exactly represent the cycling performance. Among various methods of compositional analysis, EDS (Energy Dispersive Spectroscopy) is the unique tool that can analyze the composition in the nano-sized pattern of the real device, so the reliability of the compositional analysis on the real device is up to the accuracy and repeatability of the EDS. Moreover, as it can be expected that there is a structural change in addition to the compositional change with cycles, the structural change is also analyzed by NBD (Nano Beam Diffraction) together with the compositional analysis.

In addition to the L-line X-ray that is conventionally used in the EDS analysis on GST, K-line X-ray is adopted in this study, and compared with the L-line X-ray. Although the analysis by L-line X-ray is relatively immune to the sample drift because of its shorter data collecting time due to its higher peak intensity than K-line, the peak positions of $\mathrm{Sb}$ and $\mathrm{Te}$ in the L-line X-ray are too close to distinguish between them, which often leads to the poor accuracy and repeatability of EDS analysis. However, the peaks of $\mathrm{Sb}$ and Te are placed far enough from each other that can discriminate between them in the K-line despite their low peak intensity, which results in a good accuracy and repeatability of EDS analysis. Table 1 shows the comparison of the standard deviations of five times repeated compositional analyses by both L-line and K-line X-ray for each element of GST, which reveals the better repeatability in K-line X-ray.

It was already reported by several researchers that the compositions of GST gradually change with cycles [2], which was assessed in this study by K-line X-ray as shown in Fig. 1. Sb increases gradually, but Ge and Te show the gradual decrease with cycles in the normal GST Cells. In particular, the failed cell after cycles shows the excessive compositional change.

The conventional GST is known to have the FCC (Face Centered Cubic) rocksalt structure, but it is expected that the GST structure after cycles may be changed because of the considerable compositional change. NBD (Nano Beam Diffraction) technique was adopted in this analysis because the patterned cell size was too small to use conventional beam size in the diffraction pattern analysis. Fig. 2 shows TEM images and NBD patterns of the cell before cycles, and Fig. 3 shows the cell after cycles. While there is little difference in the TEM images between the two cells, the relative position of the points in the diffraction pattern looks quite different. It is assumed that the atomic position can be deviated or tilted from the original position of FCC rocksalt structure together with the compositional change after cycles. Although the accurate indexing should be continued, it is obvious from the diffraction pattern itself that the considerable structural change after cycles was happened.

References

[1] J.-B.Park et al., J. Electrochem. Soc., 154 (3) (2007) H139.

[2] J.S. Bae et al., J. Jap. Appl. Phys., Accepted. 


\begin{tabular}{|c|c|c|c|}
\hline & Ge & $\mathrm{Sb}$ & $\mathrm{Te}$ \\
\hline \hline $\mathrm{Sb}(\mathrm{L} \alpha)-\mathrm{Te}(\mathrm{L} \alpha)$ & 3.22 & 2.46 & 2.13 \\
\hline $\mathrm{Sb}(\mathrm{K} \alpha)-\mathrm{Te}(\mathrm{K} \alpha)$ & 0.51 & 0.95 & 1.33 \\
\hline
\end{tabular}

Table 1. Standard deviation on five times repeated compositional analyses by both L-line and K-line X-ray for each element of GST. K-line was adopted for Ge on both analyses.

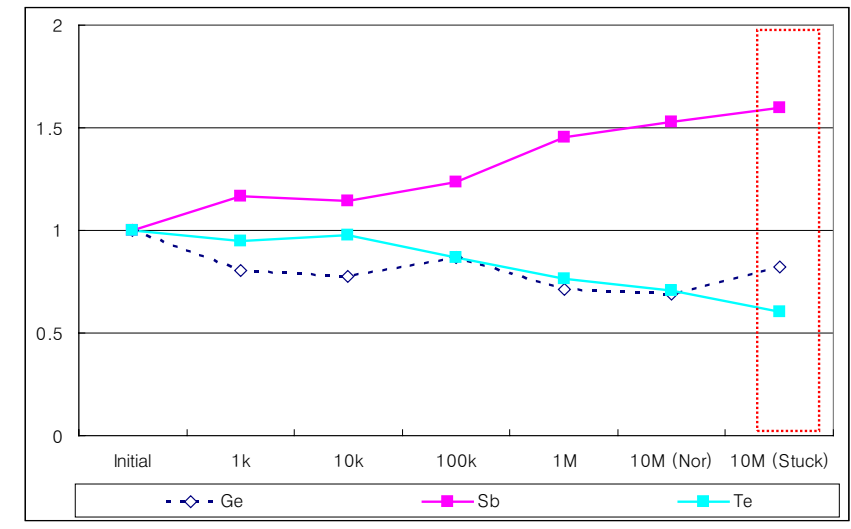

Fig. 1 Gradual change of GST composition with cycles in the normal cells. Their initial composition was normalized. Failed cell shows the excessive compositional change.

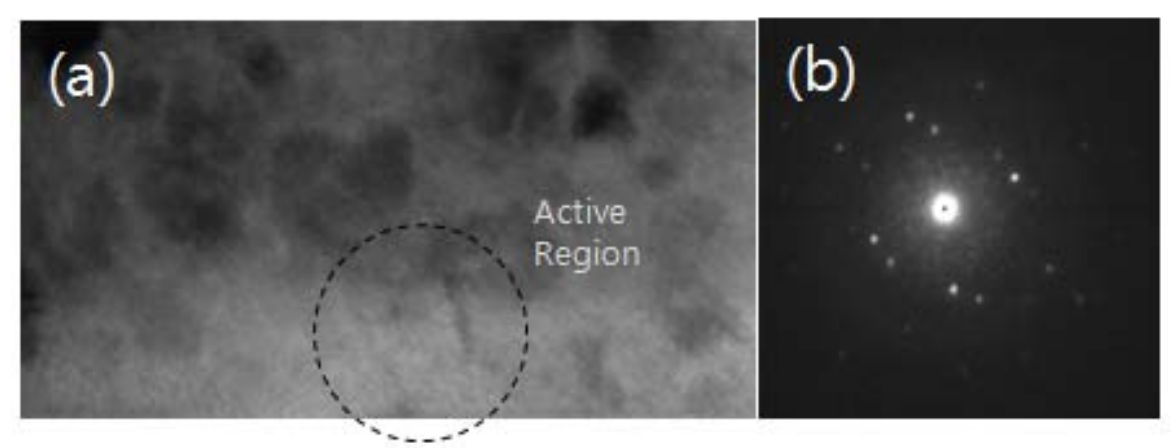

Fig. 2 Analysis on the cell before cycles (a) TEM image and (b) NBD pattern on the active region above $\mathrm{BEC}$

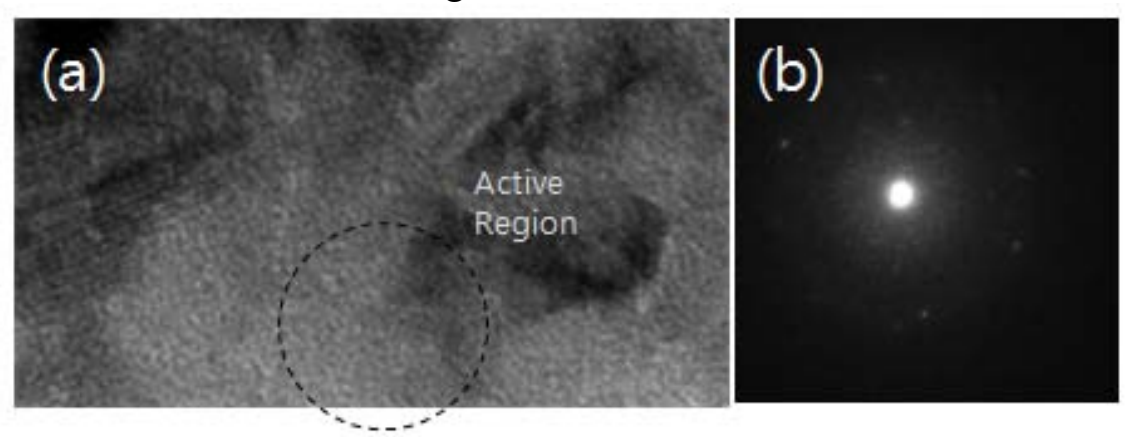

Fig. 3 Analysis on the cell after cycles (a) TEM image and (b) NBD pattern on the active region above $\mathrm{BEC}$ 